\title{
Decision Rules for the Academy Awards Versus Those for Elections
}

\author{
William V. Gehrlein, Hemant V. Kher \\ Department of Business Administration, University of Delaware, Newark, Delaware 19716-2710 \\ \{wvg@udel.edu, kher@udel.edu\}
}

\begin{abstract}
Many researchers have evaluated various decision rules to determine how well they perform in selecting winners in elections. They have established criteria to measure how well these rules perform in selecting winners with the greatest mass appeal in general elections. We evaluate such decision rules on their performance in determining winners of awards for outstanding accomplishment. We examined the procedures the Academy of Motion Picture Arts and Sciences uses to choose nominees and winners for Academy Awards. We chose this example for two reasons. First, the academy uses several decision rules to select nominees and to select the winners from the lists of final nominees. Second, Academy Awards have an enormous impact on earnings and careers. We found that decision rules that can have negative effects in elections based on mass appeal can have positive aspects in determining winners of awards for outstanding accomplishment.
\end{abstract}

Key words: games: group decisions; voting: committees.

History: This paper was refereed.

$\mathrm{W}$ inning an Academy Award, or simply being nominated for one, is considered to be a significant achievement by most members of professions associated with making movies. An award brings peer recognition, fame, prestige, power, and quite often, dramatic financial gains. Levy (2001) cited many examples of the financial benefits enjoyed as a result of being nominated for an Academy Award. For example, Faye Dunaway was paid $\$ 30,000$ for her third film, Bonnie and Clyde, for which she received her first nomination as best actress in 1967. Her pay quickly rose to $\$ 300,000$ per film, despite the fact that she did not win the award. Katharine Hepburn won the award for best actress that year.

In the last 15 years, the increased revenues from winning an Academy Award for Best Picture have been estimated at $\$ 5,000,000$ to $\$ 30,000,000$ in the United States and Canada (Levy 2001). American Beauty, which won five awards in 1999, including the best-picture award, provides a dramatic example of the impact of winning an Academy Award. The picture's gross earnings reached $\$ 130$ million by June 1, 1999, far exceeding projections. Films that win or are nominated for an Academy Award also typically obtain large payoffs when they are sold to be shown on television and when they are transferred to videocassettes and digital video disks (DVDs). In 1984, CBS reportedly paid $\$ 4.5$ million to show the 1981 winner, Chariots of Fire, and NBC paid more than $\$ 12$ million for the award nominee, On Golden Pond.

Given the enormous benefits that result from winning or being nominated for an Academy Award, we were interested in seeing how the Academy of Motion Picture Arts and Sciences nominates candidates and selects the winners. The period before the awards ceremony each year is marked by "experts" in the popular press giving extensive coverage to nominees and attempting to predict the winners. To our surprise, we found little in the literature that describes how the academy selects nominees and winners for these coveted awards. Our curiosity aroused, we wondered about

(1) The decision rules the academy uses to select nominees and winners for the different award categories and the manner in which they implemented them, and

(2) The relative strengths and weaknesses of these particular decision rules in terms of the properties of the candidates they tend to select.

We obtained the answer to our first question by visiting the academy's Web site (www3.oscars.org). In addition, academy officials answered some questions about their method of implementing of the rules. We asked for disguised election balloting data, hoping to analyze the decision rules with realistic information. However, the academy indicated that its auditing firm would not part with such data. Given this limitation, we decided to examine the extensive literature on decision-rule analysis and voting rules to gain some general insights into question (2).

\section{Nomination and Selection Procedures}

Each year the Academy of Motion Picture Arts and Sciences follows a predetermined calendar in 
qualifying films for awards, sending and receiving nomination ballots, screening nominated films, sending and receiving final ballots, and presenting the awards. The first step in the process is the nomination procedure. Here, academy members reduce the set of all possible candidates for each award category, in most cases, to a set of five final nominees. The academy determines the group of nominators differently in various categories, but each group is composed of academy members associated with the specialization of the category. The number of potential candidates available for consideration each year is so large that the academy must reduce that number to a manageable number of nominees for consideration in the final selection.

The academy stresses that its intent is to "honor outstanding achievements" in making motion pictures. This raises the issue of how one identifies outstanding achievements. All of the nominators might rate some candidate quite high, without rating him or her as the very best. Should such a candidate be considered outstanding? Such a candidate could be viewed as having mass appeal among the nominators, but should such a candidate be viewed as outstanding in the absence of a first-place ranking by a significant number of nominators? The decision rules the academy uses reflect this notion, because it typically employs decision rules that tend to favor candidates with the most first-place rankings. By selecting this approach, the academy might not recognize some candidates with mass appeal, but it reduces the chances of passing over outstanding candidates.

The popular press routinely carries articles criticizing the academy's nominations and final award selections. Much of the criticism concerns popular movies that did not win Academy Awards. However, the academy gives awards to recognize what members consider outstanding accomplishment. That does not necessarily coincide with popularity with the public. If popularity were to be used as the sole basis for recognizing outstanding achievement in the food-service industry, some well-known fast-food chains could be recognized as outstanding restaurants.

For most award categories, the academy selects nominees by using a preferential system or a variation of a weighted scoring rule, and it chooses the ultimate winners using plurality rule. For certain special awards, a committee of academy members determines nominees, and members choose the winner using a two-thirds majority rule.

\section{Nomination by the Preferential System}

The academy uses the preferential system for major award categories, including best movie, best actress, and best actor. The nominators get a list of all possible candidates in each category, and each nominator ranks his or her five most-preferred candidates from this list. The academy then uses a fairly complex procedure to sequentially select five finalists, based on the number of times nominators rank the candidates first in their preference rankings during each stage of the sequential selection process. The academy allocates each nominator's vote over the candidates in his or her rankings in different proportions during different stages of the process.

The preferential system the academy uses is a variation of a decision rule known in the literature on election procedures as single transferable vote (STV). STV has been supported as an election procedure for many years. It has been used for governmental elections in a few countries, including England, Ireland, and Malta. The Electoral Reform Society in England has a primary goal of promoting the use of STV in elections.

Example 1 illustrates how the academy's preferential system works. We want to obtain three nominees from a list of 12 possible candidates: A, B, C, .., L. We have 540 ballots from nominators, each showing the nominator's ranking of his or her five mostpreferred candidates from the list of 12 possible nominees. A total of 95,040 possible five-candidate rankings could represent any nominator's preferences. However, for the sake of simplicity, we assume each nominator has ranked preferences on his or her five most-preferred candidates that fall into one of the 12 preference categories in Table 1. In this example, 30 nominators' ballots have the same response as those in Preference Category \#1. A is the most-preferred candidate, followed in decreasing preference by $\mathrm{B}, \mathrm{K}$, $\mathrm{E}$, and D. Each unlisted candidate of the 12 possible candidates is less preferred than every candidate ranked on the ballot.

Under the preferential system, we try to add candidates receiving the most first-place votes to the final list of nominees in this initial step. We enter candidates to the final list if they receive enough first-place votes to meet a cutoff that is known in the literature as the Droop quota (Black 1958, p. 73). For a general situation in which $n$ voters are selecting $m$ nominees, the Droop quota is calculated as $n /(m+1)+1$. For our

Preference category

\begin{tabular}{cccccccccccc}
\hline 11 & $\# 2$ & $\# 3$ & $\# 4$ & $\# 5$ & $\# 6$ & $\# 7$ & $\# 8$ & $\# 9$ & $\# 10$ & $\# 11$ & $\# 12$ \\
\hline A & B & B & C & C & C & D & D & K & F & J & L \\
B & K & G & H & K & B & A & B & E & K & D & A \\
K & A & J & B & B & D & J & I & G & I & B & B \\
E & E & F & F & A & A & G & J & B & B & C & D \\
D & D & D & D & D & E & F & C & D & D & A & G \\
30 & 30 & 72 & 90 & 68 & 114 & 22 & 25 & 49 & 22 & 17 & 1
\end{tabular}

Table 1: We show the voter preferences at Stage 1 in Example 1. 
particular example, the Droop quota is $540 /(3+1)+$ $1=136$.

We can describe the logic behind the calculation of the Droop quota in terms of our example. The candidates that we select as final nominees should be those with the most first-place votes from the 540 nominators, while meeting some minimum number. This minimum number is the Droop quota, 136. All candidates with at least 136 first-place votes make the list of final candidates in this initial stage, if there are any such candidates. We could have as many as three candidates (but not four) with at least 136 first-place votes with 540 nominators. Thus, we cannot add too many candidates to the final list in this stage when we use the Droop quota.

Candidate $\mathrm{C}$ has the most first-place votes with 272, so we list candidate $C$ as a final nominee. We select no other candidates at this stage, because none have enough first-place votes to meet the Droop quota. Although $C$ needed only 136 votes to be selected, it received twice that number. As a result, each ballot with $C$ ranked in first place will be charged only one half of its vote to elect $C$, with the remaining half vote being carried forward to count for the second-ranked candidate. The intent is to encourage nominators to give their true preference rankings on ballots by limiting the penalty for voting for a heavily favored candidate. Nominators can rank their true preferences without fearing that they are wasting their entire vote on their most-preferred candidate, when that candidate is a guaranteed winner.

In the second stage, we remove candidate $C$ from all preference rankings because it is a final nominee. We also remove candidates E, G, H, and I according to the academy's preferential system because they received no first-place votes. The reasoning here is that if no nominator thought a candidate was the very best for the category, it should not be considered for nomination. We also remove candidate $\mathrm{L}$ from the rankings, because it is the favorite of only a single nominator and it appears in the rankings of no other nominator. This single nominator would effectively be ignored during the entire voting process if $L$ were not removed, and $\mathrm{L}$ could not be a final nominee in this case anyway.

After removing candidates and assessing the onehalf votes for ballots in Preference Categories \#4, \#5, and \#6 to elect $C$, we have the rankings on the remaining candidates that are shown in Table 2.

We have reduced the total number of ballots to 404 by eliminating the 136 votes used to elect $C$. We continue to use the Droop quota to select the remaining nominees. Candidate B now has the most first-place votes on the modified ballots with 204 votes, which exceeds the Droop quota of 136. We therefore enter candidate B as a final candidate. However, B has
Preference category

\begin{tabular}{lccccccccccc}
\hline$\# 1$ & $\# 2$ & $\# 3$ & $\# 4$ & $\# 5$ & $\# 6$ & $\# 7$ & $\# 8$ & $\# 9$ & $\# 10$ & $\# 11$ & $\# 12$ \\
\hline A & B & B & B & K & B & D & D & K & F & J & A \\
B & K & J & F & B & D & A & B & B & K & D & B \\
K & A & F & D & A & A & J & J & D & B & B & D \\
D & D & D & - & D & - & F & - & - & D & A & - \\
- & - & - & - & - & - & - & - & - & - & - & - \\
30 & 30 & 72 & 45 & 34 & 57 & 22 & 25 & 49 & 22 & 17 & 1
\end{tabular}

Table 2: We show the voter preferences at Stage 2 in Example 1.

150 percent of the votes needed for nomination, so we will assess each ballot with B ranked in first place as only two-thirds of a vote and elect $B$. The remaining one-third of a vote will be carried forward to the second-ranked candidates on the modified ballots on which B is ranked first. After selecting B as a final nominee, removing $B$ from the rankings and removing the partial votes for nomination from ballots in Preference Categories \#2, \#3, \#4, and \#6, we have the modified rankings in Table 3.

Candidate $\mathrm{K}$ now has the most first-place votes, with 93 votes, but this number does not meet the Droop quota. We continue to sequentially eliminate the candidate with the fewest first-place votes in each stage until we find some candidate with enough firstplace votes to exceed the Droop quota. After sequentially eliminating $\mathrm{A}$ and $\mathrm{F}$, we find that candidate $\mathrm{K}$ finally has 145 votes-enough to be selected as a final nominee.

\section{Properties of the Preferential System}

The ballots in Preference Categories \#7 and \#8 never come into play in Example 1. The nominators' mostpreferred candidate, $\mathrm{D}$, in these categories is the only one listed on all 540 original ballots. However, D was not ranked high enough in the ballots in the other preference categories to reach first place in the preference rankings for nomination during the sequential elimination process. Clearly, this procedure does not recognize candidates for being listed on a lot of ballots, but for being ranked first on ballots.

Preference category

\begin{tabular}{cccccccccccc}
\hline$\# 1$ & $\# 2$ & $\# 3$ & $\# 4$ & $\# 5$ & $\# 6$ & $\# 7$ & $\# 8$ & $\# 9$ & $\# 10$ & $\# 11$ & $\# 12$ \\
\hline $\mathrm{A}$ & $\mathrm{K}$ & $\mathrm{J}$ & $\mathrm{F}$ & $\mathrm{K}$ & $\mathrm{D}$ & $\mathrm{D}$ & $\mathrm{D}$ & $\mathrm{K}$ & $\mathrm{F}$ & $\mathrm{J}$ & $\mathrm{A}$ \\
$\mathrm{K}$ & $\mathrm{A}$ & $\mathrm{F}$ & $\mathrm{D}$ & $\mathrm{A}$ & $\mathrm{A}$ & $\mathrm{A}$ & $\mathrm{J}$ & $\mathrm{D}$ & $\mathrm{K}$ & $\mathrm{D}$ & $\mathrm{D}$ \\
$\mathrm{D}$ & $\mathrm{D}$ & $\mathrm{D}$ & - & $\mathrm{D}$ & - & $\mathrm{J}$ & - & - & $\mathrm{D}$ & $\mathrm{A}$ & - \\
- & - & - & - & - & - & $\mathrm{F}$ & - & - & - & - & - \\
- & - & - & - & - & - & - & - & - & - & - & - \\
30 & 10 & 24 & 15 & 34 & 19 & 22 & 25 & 49 & 22 & 17 & 1
\end{tabular}

Table 3: We show the voter preferences at Stage 3 in Example 1. 
An advantage of STV-like procedures lies in the context of strategic misrepresentation of preferences. In Example 1, we demonstrated the importance of the number of first-place ranks in gaining inclusion on the nominee list when STV is used. Voters might abuse this system by giving their preferred candidate very high ranks, while giving the leading rival the lowest possible rank to try to alter the outcome of the nomination process. Bartholdi and Orlin (1991) concluded that the complexity of STV makes it practically immune to strategic misrepresentation, despite the theoretical possibility that a nominator's misrepresentation might alter the outcome.

A classic criterion in election procedures is the propensity of decision rules to elect the Condorcet winner. A Condorcet winner is a candidate who would beat every other candidate in a series of simple majority-rule votes on pairs of candidates. A Condorcet winner does not necessarily exist, but designers of an election procedure might want to select it when one does exist, because a majority of voters would oppose replacing that winner with any other candidate. This criterion is biased toward selecting candidates with mass appeal. Because candidate $C$ received 272 first-place rankings from the 540 nominators in Example 1, it is the Condorcet winner, and the preferential system included it in the final list of nominees. The procedure violates the spirit of the Condorcet criterion in its placement of candidates D and K. Candidate K is ranked ahead of D by only 199 of the 540 nominators. So D would win over K by majority rule on the pair of candidates, but $\mathrm{K}$ makes the final list of nominees, while $\mathrm{D}$ does not.

Example 2 is an extreme example in which the preferential system does not select the Condorcet winner. Suppose that we are using the preferential system to select three nominees from a list of 12 possible candidates, as we did in Example 1. We still have 540 nominators who rank five candidates on their ballots, but this time their preferences all fall into just three categories of preference types as shown in Table 4.

The Droop quota is still 136, as in Example 1. We select candidates $\mathrm{A}, \mathrm{B}$, and $\mathrm{C}$ as the final nominees because they each have 180 first-place votes, even

Preference category

\begin{tabular}{lcc}
\hline$\# 1$ & $\# 2$ & $\# 3$ \\
\hline A & B & C \\
D & D & D \\
B & C & A \\
C & A & B \\
E & F & G \\
180 & 180 & 180
\end{tabular}

Table 4: These are the voter preferences in Example 2. though $\mathrm{D}$ is the Condorcet winner because it would beat A, B, or C by a margin of 360 to 180 in a direct pairwise comparison, while beating all other candidates 540 to 0 . Thus, we have a situation in which two-thirds of the voters would rather have selected D than any of the three final nominees that were picked. Again, the reason for this outcome is that D does not rank in first place on enough rankings.

Lynch (2001) reports that a situation with a nearly equal division of first-place votes like that in Example 2 happened in 1992. Some believe that Marisa Tomei won by a fluke in the best-supporting-actress category for her role in My Cousin Vinnie, because the academy voters were equally split among the five nominees for the category. In this situation the winner would not tend to get widespread recognition after winning. After winning the award, Ms. Tomei was quoted as saying "I didn't feel like a success...." However, the possibility of such an outcome would seem to be rare.

\section{Context Appropriateness of the Preferential System} STV has been used in political elections. While STV has some nice properties, it can result in the election of individually strong factions that form politically unstable parliaments or committees. In an election, we would like to be able to count on candidates with widespread appeal, like Condorcet winners, to deliver stable political situations. However, our two examples demonstrated that STV does not necessarily pick candidates of this type. Thus, in a general election, the use of something like the preferential system could cause a troublesome situation if the five final nominees were to form a ruling committee. If each of the committee members had very strong support from a small faction in the nominating group, the factions and nominees would likely have very different interests. The members of the ruling committee might then be very antagonistic and the committee unstable. The five nominees for the awards, however, are not expected to work together in any context, so the criticism becomes a moot point in such situations.

Coy (2000) quotes Steven Brams as being critical of STV in the context of general elections and as supporting the use of approval voting. With approval voting, each nominator votes to approve or disapprove of each candidate. The candidate receiving the greatest number of approval votes is then selected. To win under approval voting, candidates must broaden their bases of appeal to become centrist candidates to be considered acceptable to the greatest number of voters.

Saari (2001) is critical of the use of approval voting in general elections. He argues that such an approach tends to produce many possible winners, depending on the number of candidates each nominator 
would consider acceptable. In Example 1, each of the 540 nominators could vote for just his or her mostpreferred candidate, or for his or her two, three, four, or five most-preferred candidates. The number of voting outcomes would be huge, with potentially different winners in the different scenarios, based solely on the number of candidates each voter considered acceptable.

Using STV might not be appropriate for general elections in which popular centrist candidates would seem like good choices for maintaining political stability. However, using STV seems appropriate to the academy's stated goal of recognizing outstanding performance in filmmaking, so that any film or individual that gets very strong support from a large segment of the membership will be included as a final nominee. The use of this procedure also explains the occasional inclusion of nominees that outsiders might view as odd selections.

\section{Nomination by a Weighted-Scoring Rule}

Under a pure weighted-scoring rule, each nominator would rank all $k$ possible candidates under consideration. Then, each nominator would give a score of $w_{1}$ to his or her most-preferred candidate, $w_{2}$ to his or her second-most-preferred candidate, and so on, with $w_{1} \geq w_{2} \geq \cdots \geq w_{k}$. The total score for each candidate is then the sum of scores accumulated over the preferences of all nominators. Candidates would be included on the final list of nominees, starting with the one with the greatest total score. Using such a procedure would be impractical for the Academy Awards, because it would be unreasonable to expect nominators to rank all of the many possible candidates.

The academy uses a variation of this system to select the final nominees for some award categories, including best short documentary, best feature-length documentary, best animated short film, and best liveaction short film. While these awards have less public visibility than such awards as best movie, winning in these categories still has great impact. Steve Jobs, chairman and CEO of Pixar Animation Studios, writes at length in the firm's 1997 annual report about the importance of winning the award for best animated short film for its entry, Geri's Game.

The modified weighted-scoring rules the academy uses are fairly simple. Nominators rate each candidate on a numerical scale of integer values ranging from 5 to 10, or 6 to 10, depending on the category. Those films with an average rating above a fixed minimum, 7.5 or 8.5 respectively, are then eligible for nomination. There can be no fewer than three and no more than five nominees in each category. This rule is a variation of a weighted-scoring rule in that nominators effectively segment the large number of candidates into five or six groups, for 6 to 10 scores or 5 to 10 scores respectively, and rank the groups. Then, candidates in the top-ranked group get 10 points each, candidates in the next highest group get nine points each, and so on. The scores assigned to groups within the rankings of these six groups of candidates $(10,9$, $8,7,6,5)$, change in a linearly decreasing fashion. This weighting scheme is the basis of the pure weightedscoring rule known as the Borda rule, which has been used for years. McLean and London (1990) note that Nicolas Cusanus suggested a pure weighted-scoring rule exactly like it in the 1300 s.

\section{Properties of Weighted-Scoring Rules}

Benoit (1992) analyzed the specific non-Borda weighted-scoring rule that the Baseball Writers Association of America uses to select winners for the most-valuable-player (MVP) awards for major league baseball. Voters rank their 10 most-preferred nominees in each of the American and National League divisions. Candidates receive weights of $(14,9,8,7$, $6,5,4,3,2,1)$ according to the reported rankings. The substantially greater weight for a first-place vote rewards candidates that voters view as the very best and gives less recognition to lower place rankings. Benoit (1992) was interested in the likelihood that slight modifications to the weights in this scoring rule might alter the final selection of the most valuable player in each division, given the actual voter rankings reported from 1943 through 1989. In a number of cases, a particular candidate had nearly unanimous support as first-place choice, so modifying the scoring rule would not have changed the outcome. However, in some cases the outcome was sensitive to the weights used. For example, Mickey Mantle won the most-valuable-player award three times. He could have won the award in two additional years with a different weighted-scoring rule. Clearly, the weights used in a weighted-scoring rule can be critical.

We can see the degree to which the selected weights can affect the outcome by analyzing the nominators' preferences in Example 1, when we assume that they rank candidates on the 10 to 5 scale. We make the same assumption about the preference rankings in Example 1 that we did when considering the preferential system. For example, nominators in Preference Category \#1 will assign 10 points to $A$, nine points to $B$, eight points to $K$, seven points to $E$, six points to $D$, and five points each to all the remaining candidates that are not ranked. As before, we assume that all candidates that are not listed in a nominator's preference ranking are less preferred than each of the ranked candidates.

The total score under the weighting scheme (10, $9,8,7,6,5)$ results in the selection of three 
candidates: B (4,556 points), C (4,119 points), and D $(3,708$ points). Thus, this procedure would produce a different final list of nominees than the preferential system that selected candidates B, C, and K. We obtain different outcomes with different weights. With weights $(14,12,10,7,6,5)$ the three final candidates would still be B (5,823 points), C (5,207 points), and $\mathrm{D}(4,175$ points). A slight change in the weighting scheme to $(14,13,10,7,6,5)$ produces final nominees B (5,992 points), C (5,207 points), and $K$ (4,251 points). Using the weighting scheme $(6,6,6,6,5,5)$ produces final nominees $B(3,218$ points), $C$ ( 2,989 points), and A (2,965 points).

The preferences in Example 1 include so many firstand second-place rankings for $B$ and $C$ that it would be difficult to exclude them from the final set of three candidates. However, the third candidate in the set could be A, D, or $\mathrm{K}$ depending on what weights we use. Candidate $\mathrm{D}$ would beat both $\mathrm{B}$ and $\mathrm{C}$ with the weighting scheme $(6,6,6,6,6,5)$ if we sought a single finalist instead of three. Saari (1992) presented an analysis to show that this general phenomenon is to be expected. That is, for any given set of nominators' preference rankings, we should anticipate the possibility that different weighting schemes will produce different winners.

Merlin et al. (2000) generalized some results from Saari (1994) to obtain the necessary conditions on a set of voters' preferences for all weighted-scoring rules to produce the same winner. Let Rule $C_{j}$ denote the weighted-scoring rule that assigns a weight of one to the first $j$ positions and a weight of zero to all other positions. Then, all weighted-scoring rules on $k$ candidates will select the same winner if the same winner is selected by all Rule $C_{j}$ for $j=1,2,3, \ldots, k-1$. They obtain results that indicate that this probability is relatively large for three-candidate elections. However, Gehrlein and Lepelley (2000) extended these results to situations with more than three candidates and found that the probability that all weighted-scoring rules elect the same winner decreases dramatically as the number of candidates increases beyond three. The high degree of complexity of STV makes it virtually impossible to develop any representation for the probability that it will select the same winner as a weighted-scoring rule.

Saari (2001, pp. 149-151) examined variations of voting procedures that are somewhat more like the actual procedure the academy uses than we suggested in our analysis of the implementation of weightedscoring rules on Example 1 data. These variations allow nominators to select as many acceptable candidates as they wish to vote for, and these procedures are referred to as truncated voting rules. If a nominator selects $q$ candidates as worthy of receiving votes, then these acceptable candidates would be given the associated scores $w_{1}, w_{2}, \ldots, w_{q}$. As in approval voting, one can expect a wide range of potential voting outcomes, because the outcomes are a function of the number of candidates each nominator votes for. The procedure the academy uses is even more flexible than these truncated voting rules, because weights can be skipped in the point assignments. That is, a nominator might assign a score of 10 to some candidates and a score of 8 to some candidates, without assigning a score of 9 to any. We would expect even more different voting outcomes with such a voting rule. However, with such a large number of potential candidates, the nominators would probably use all possible points on the range of allowable scores to reflect degrees of preference.

The use of weights for scoring rules in agreement with a strict Borda rule has been examined extensively since Borda (1989) formally proposed it as a voting procedure for the French Academy of Sciences, and it has been shown to have some very nice properties. If the nominators doing the rankings tend to have preferences on candidates that are statistically independent, the Borda rule is the strict weighted-scoring rule that will tend to maximize the likelihood that Condorcet winners are included in the set of nominees (Gehrlein and Fishburn 1978). Saari (1989) considered negative paradoxical voting outcomes from using strict weighted-scoring rules and showed that the Borda rule uniquely minimizes the probability of such outcomes. Given this background, the academy seems justified in selecting the Borda rule from all possible weighted-scoring rules.

A drawback of the variation of the Borda rule the academy uses is that it can be subject to problems with strategic misrepresentations of preferences. That is, nominators can gain advantages for their favorite candidates by giving them high scores and giving very low scores to their major contenders. We see this possibility in Example 1 with nominators who give scores between 5 and 10 to the 12 possible candidates.

Candidates $\mathrm{B}$ and $\mathrm{C}$ are the leading candidates under a wide range of selection procedures. Of the 540 nominators, 272 rank $C$ as their most-preferred candidate, and they rank $\mathrm{B}$ as second or third. If these nominators misrepresented their preferences by giving a score of 10 to $\mathrm{C}$ and a score of 5 to $\mathrm{B}$, the supporters of $C$ would make it impossible for $C$ 's primary opponent, candidate $\mathrm{B}$, to have an average score greater than 7.5. Candidate B would not be able to obtain the minimum required average score for inclusion in the list of final nominees, regardless of how his or her supporters voted.

Gibbard (1973) and Satterthwaite (1975) partly minimize the significance of the issue of strategic manipulation by showing that voters can manipulate 
the outcomes of effectively every election procedure by strategically misrepresenting their preferences. Favardin et al. (2002) examined the probability that various voting procedures, including the pure weighted-scoring rule using the Borda rule, could be manipulated by individuals and by coalitions in three-candidate elections. They found that the probability that an individual might manipulate an election outcome is negligible for a large number of voters. However, the probability that a coalition of voters might manipulate an election outcome is higher for the Borda rule than for other common voting procedures, even for large numbers of voters.

Because the weighted-scoring rules have the potential to be manipulated seems to condemn variations of the Borda rule. However, Slinko (2002) provided evidence that the academy need be little concerned about strategic manipulation because of the large number of voters. Slinko (2002) used the same assumptions about voter preferences as Favardin et al. (2002) to show that the probability $L(m, n, k)$ that a coalition of $k$ voters, of $n$ total voters, can manipulate a weightedscoring rule with $m$ candidates has $L(m, n, k) \leq$ $D_{m}(k / n)$. Here, $D_{m}$ is a finite constant that is a function of $m$ and $k$, but not of $n$. As a result, only a very large coalition of voters of a very large number of decision makers could possibly change the election outcome of a weighted-scoring rule by misrepresenting their preferences.

The Borda rule has some good properties, and it would be useful in determining nominees for Academy Awards, subject to the review committees' maintenance of stringent standards of integrity to minimize the possibility of misrepresentation of preferences. Rodman (1989) suggested that the reviewing committees take their charge very seriously. Evidence exists to determine how strict the review committees have been in imposing standards. The number of final nominees in the four categories for best documentary ranges from three to five. If the review committees graded easily, we would expect five-nominee cases quite frequently. That is, the committees might think that nominations are available to be given out, they are important to people receiving them, so why not award them? For the 25-year period 1978 through 2002, the data in Table 5 indicates that the reviewing committees are not giving arbitrarily high marks, or the number of five-nominee cases would be greater, particularly in the short-film categories.

\section{The Final-Selection Procedure}

During the nomination stage, the academy uses decision rules that tend to select candidates based on outstanding accomplishment rather than mass appeal. Does it maintain this sentiment in choosing final winners? At the final stage, voting is open to all active

\begin{tabular}{lrcr} 
& \multicolumn{3}{c}{ Number of nominees } \\
\cline { 2 - 4 } Award category & 3 & 4 & 5 \\
\hline Documentary—short & 5 & 0 & 20 \\
Documentary—-feature & 0 & 0 & 25 \\
Short film—animated & 15 & 2 & 8 \\
Short film—live action & 10 & 1 & 14
\end{tabular}

Table 5: The number of nominees in the Documentary-Short film categories for 1978 through 2002 range between three and five. For the Documentary-Short category award, there were five years with three nominees, zero years with four nominees, and 20 years with five nominees. The numbers for the other categories may be interpreted similarly.

and lifetime academy members, and it is done using the plurality rule in each category. Each voter casts a vote for his or her preferred candidate in each category, and the candidate who receives the most votes wins. All members of the academy can be assumed to know what constitutes outstanding performance in the various categories.

The academy uses a decision rule that emphasizes the number of first-place votes that candidates receive. Gehrlein and Fishburn (1978) showed that when voters' preferences are statistically independent, the plurality rule is the weighted-scoring rule least likely to select the Condorcet winner. Thus, the tradeoff of recognizing excellence as opposed to mass appeal continues. On the other hand, Gehrlein (2003) showed that weighted-scoring rules that are more like the plurality rule than the Borda rule tend to select the Condorcet winner when voter preferences are dependent in the statistical sense. Because voters do not rank the candidates in the final stage of the election, we could not examine the dependence among voters' preference rankings, even if the election results were available.

Lyman (2002) quotes Steven Brams as strongly supporting the use of approval voting in the final election stage for determining winners of Academy Awards. Brams argues that the plurality voting procedure the academy uses might be less likely than approval voting to select a Condorcet winner. A Condorcet winner may not have won the award for best movie in 1976, when Rocky was selected over All the President's Men, Bound for Glory, Network, and Taxi Driver. Brams argues that under pairwise comparison Rocky would have lost to some of the other candidates, and that the use of approval voting would have prevented Rocky's winning. However, it is impossible to prove this claim, or even if a Condorcet winner existed at all, without knowing the preference rankings of the voting academy members on the five candidates.

In discussing the use of approval voting during the nomination phase, we concluded that the academy might choose to use their preferential system to determine outstanding candidates, rather than mass appeal 
or centrist candidates. However, Brams' argument is more convincing for the final election stage. The academy may want to select the candidate with the greatest mass appeal from the final list of candidates that have already been identified as outstanding.

\section{The Two-Thirds Majority Rule}

The academy's board of governors gives out three awards for excellence in various aspects of filmmaking:

- The Thalberg Award for consistency in highquality work as a movie producer,

- The Hersholt Award for humanitarian efforts bringing credit to the industry, and

- The Sawyer Award for technological contributions to the movie industry.

These awards are not honorary awards, like those for lifetime achievement. The board first conducts elections to determine a leading prospect for each award. It then requires a two-thirds majority vote to determine whether to give each of the leading prospects the associated award. This type of rule is called a super-majority rule.

\section{Properties of Super-Majority Rules}

The major drawback is that it can be hard to get a winner by super majority. Colomer and McLean (1998) cited instances in which the College of Cardinals was deadlocked for months while trying to elect a new pope under a two-thirds majority rule during the 1200s. In each instance, no candidate could garner the votes necessary to win. In 1241, the civil administration of Rome confined the College under police guard and forced a decision by threatening to exhume the dead pope and publicly display his corpse in full regalia. Another deliberation started in 1268, with the cardinals housed in a palace. After a two-year deadlock, the public besieged the palace, removed the roof from the building, and allowed only bread and water to be taken in. The deadlock lasted for 34 months. The prolonged election periods brought about a great deal of instability. However, stability returned until the next election once the cardinals came to a consensus and elected a new pope.

Coggins and Perali (1998) discussed a prolonged process that was used in Venice to elect the doge, or duke. The position was a lifetime appointment, and between the death of one doge and the election of the next, the government came to a standstill. In a series of stages employing lotteries and super-majority voting, the populace ultimately selected a voting body of 41 members. When 25 of the 41 members agreed on a candidate, for a 61 percent super majority, the doge was elected. Venice used the system for about 500 years, enduring many periods when the government was at a standstill. However, the process is credited with creating the very stable overall political climate of the period.

The academy's board of governors does not face periods of instability if no candidate gets two-thirds of the voters' support; it can simply not make the award that year. However, we would not expect the academy to withhold awards frequently, given the two-thirds majority requirement, if the governors were acting as easy graders who are inclined to give awards simply because they are available. We determine if this has been the case by examining existing records.

From 1978 through 2002, the academy gave out the Thalberg Award 12 times and the Hersholt Award 13 times. The Sawyer Award has existed since 1981 and has been given out 15 times. The board of governors has apparently been quite strict in their evaluations for these awards, giving them on only 56 percent of the possible instances.

\section{Conclusions}

Our analysis shows that the academy has generally chosen decision rules that select nominees based on outstanding achievement, rather than mass appeal. The objectives in a general election differ from those of the academy. While the STV rule helps the academy identify outstanding performers, in a general election it could elect individuals with strong, contrasting personalities and agendas. Such a situation could lead to political turmoil. Similarly, the super-majority rule helps the academy to be very selective in choosing recipients for certain awards, while in a political context it may prolong the process of choosing a candidate. History suggests, however, that the prolonged search time encourages overall political stability. One must consider such factors before choosing decision rules.

\section{Acknowledgments}

We gratefully acknowledge the assistance provided by the Academy of Motion Picture Arts and Sciences, particularly by Richard Miller and Bruce Davis. We thank Burton Abrams for suggesting that we analyze the academy's decision rules and for trying to obtain information about the academy's nomination process. Two anonymous reviewers made many helpful suggestions for improving earlier versions of this paper. Any errors in the manuscript are solely our responsibility.

\section{References}

Bartholdi, J. J., J. B. Orlin. 1991. Single transferable vote resists strategic voting. Soc. Choice Welfare 8(4) 341-354.

Benoit, J. P. 1992. Scoring reversals: A major league dilemma. Soc. Choice Welfare 9(2) 89-97.

Black, D. 1958. The Theory of Committees and Elections. Cambridge University Press, Cambridge, U.K. 
Borda, J. de. 1784. A paper on elections by ballot. F. Sommerlad, I. McLean. 1989. The political theory of Condorcet. Working paper, University of Oxford, Oxford, U.K.

Coggins, J. S., C. F. Perali. 1998. Sixty-four percent majority rule in ducal Venice: Voting for the doge. Public Choice 97(4) 709-723.

Colomer, J. M., I. McLean. 1998. Electing popes: Approval balloting and qualified majority rule. J. Interdisciplinary History 29(1) $1-22$.

Coy, P. 2000. Consider the alternatives. Bus. Week 3710(December 4) 126.

Favardin, P., D. Lepelley, J. Serais. 2002. Borda rule, Copeland method and strategic manipulation. Rev. Econom. Design 7(2) 213-228.

Gehrlein, W. V. 2003. Weighted scoring rules that maximize Condorcet efficiency. M. Sertel, S. Koray, eds. Adv. Econom. Design. Springer, Berlin, 53-64.

Gehrlein, W. V., P. C. Fishburn. 1978. Coincidence probabilities for simple majority and positional voting rules. Soc. Sci. Res. 7(3) 272-283.

Gehrlein, W. V., D. Lepelley. 2000. The probability that all weighted scoring rules elect the same winner. Econom. Lett. 66(2) 191-197.

Gibbard, A. 1973. Manipulation of voting schemes: A general result. Econometrica 41(4) 587-601.

Levy, E. 2001. OSCAR Fever: The History and Politics of the Academy Awards. Continuum International Publishing Group, New York.
Lyman, R. 2002. French twist: A fair way to pick Oscars? New York Times (March 17).

Lynch, L. 2001. Who's news. USA Weekend (February 9) 4.

McLean, I., J. London. 1990. The Borda and Condorcet principles: Three medieval applications. Soc. Choice Welfare $\mathbf{7 ( 2 )}$ 99-108.

Merlin, V., M. Tataru, F. Valognes. 2000. On the probability that all the rules select the same winner. J. Math. Econom. 33(2) 183-207.

Rodman, H. A. 1989. The five nominees and how they grew. Film Comment 25(2) 8.

Saari, D. G. 1989. A dictionary of voting paradoxes. J. Econom. Theory 48(2) 443-475.

Saari, D. G. 1992. Millions of election outcomes from a single profile. Soc. Choice Welfare 9(4) 277-306.

Saari, D. G. 1994. Geometry of Voting. Springer, New York.

Saari, D. G. 2001. Chaotic Elections! A Mathematician Looks at Voting. American Mathematical Society, University of CaliforniaIrvine, Irvine, CA.

Satterthwaite, M. 1975. Strategy-proofness and Arrow's condition. J. Econom. Theory 10(2) 198-217.

Slinko, A. 2002. On asymptotic coalitional strategy-proofness of social choice rules under the IAC assumption. Report series 483, Department of Mathematics, University of Auckland, Auckland, New Zealand.

Sommerlad, F., I. McLean. 1989. The political theory of Condorcet. Working paper, University of Oxford, Oxford, U.K. 